\title{
Influence des voisins sur le développement des espèces locales introduites dans les formations naturelles soudaniennes et guinéennes du Bénin
}

\author{
Laurent DJODJOUWIN *, Romain GLELE KAKAÏ et Brice SINSIN \\ Laboratoire d'Ecologie appliquée, Faculté des Sciences Agronomiques, Université d'Abomey-Calavi, 01 BP \\ 526 Cotonou, Bénin. \\ *Auteur correspondant, E-mail: djodjou@yahoo.fr; Tél. ++22995 509104
}

\section{RESUME}

L'objectif de l'étude est d'analyser l'influence des voisins sur la croissance des arbres des 4 espèces suivantes introduites dans les formations naturelles dégradées du Bénin: Terminalia superba; Holoptelea grandis; Khaya grandifoliola; Khaya senegalensis. Ainsi, 170 et 31 placettes circulaires de $10 \mathrm{~m}$ de rayon sont aléatoirement installées dans les plantations d'enrichissement de la forêt classée de la Lama et des galeries forestières de Bassila avec pour centre les individus des espèces introduites. Dans chaque placette, le diamètre, la hauteur et la distance séparant l'espèce introduite de ses voisins sont mesurés. Le statut social de dominé, codominant et dominant des arbres est apprécié. Une analyse en composantes principales est effectuée sur les paramètres dendrométriques liés aux compétiteurs et a permis d'extraire les axes principaux qui sont ensuite corrélés avec les paramètres dendrométriques et sociologiques des individus centraux afin d'analyser l'influence des compétiteurs sur le développement des espèces introduites. Les résultats indiquaient que dans les forêts enrichies de la zone guinéenne, le nombre élevé de compétiteurs autour des individus introduits et leur hauteur et diamètre élevés ont maintenu certaines espèces comme Holoptelea grandis, Khaya grandifoliola et Khaya senegalensis dans la strate inférieure. Concernant Terminalia superba, la hauteur, le diamètre et la densité élevés des compétiteurs autour, ont influé positivement sur sa croissance en hauteur. Dans les forêts enrichies de la zone soudano-guinéenne, les compétiteurs n'avaient pas d'influence significative sur la croissance de Terminalia superba et de Khaya senegalensis mais ils affectaient négativement la croissance en diamètre et positivement la croissance en hauteur de Khaya grandifoliola. Les voisins influençaient donc le développement des espèces introduites à travers l'ombrage qu'ils leurs créaient, ce qui doit être pris en compte dans la gestion des formations naturelles enrichies.

(C) 2011 International Formulae Group. All rights reserved.

Mots clés: Formations naturelles, enrichissement, influence des voisins, zone climatique, Bénin.

\section{INTRODUCTION}

La dynamique et la production des peuplements forestiers naturels ont fait l'objet de préoccupations importantes de la communauté scientifique internationale depuis plusieurs décennies. En effet, face à la disparition accélérée des forêts tropicales
(Sinsin et al., 2002), il s'est avéré urgent d'orienter les recherches en sciences forestières sur les possibilités de reconstitution $\mathrm{du}$ potentiel sur pied après l'exploitation (Schmitt et Barriteau, 1990). Le Bénin a choisi entre autres possibilités de reconstitution, l'introduction dans les 
formations naturelles dégradées des espèces locales adaptées à travers des plantations d'enrichissement. Les plantations d'enrichissement consistent à introduire dans un milieu forestier plus ou moins transformé, des essences commerciales qui deviennent à terme, l'essentiel de la production en bois tout en assurant un rétablissement écologique de la forêt (Dupuy, 1992). L'aménagement forestier de ces formations naturelles enrichies nécessite l'étude des caractéristiques structurales et de la dynamique individuelle des arbres des peuplements. En effet, l'un des aspects importants de la dynamique forestière est l'accroissement individuel et général du diamètre et de la surface terrière des arbres. Aussi, l'étude de la dynamique forestière devra-t-elle, pour donner de bons résultats exploitables par les gestionnaires, s'en tenir aux peuplements stratifiés selon des critères phytoécologiques et dendrométriques (Nguyen-The et al., 1999). Pour ces peuplements stratifiés, il est utile d'étudier les liaisons entre les productivités dynamiques mesurées des produits mélangés ou classés par catégorie, des facteurs phytoécologiques tels que le climat, le sol et la structure de la végétation et des facteurs dendrométriques comme la surface terrière, l'arbre moyen, la hauteur et la dimension moyenne des cimes, à partir des outils d'analyse de données pertinentes. Par ailleurs, la croissance d'un arbre peut être favorisée ou non par la présence de ses voisins (Otto, 1997). L'objectif de l'étude est de contribuer à un meilleur dispositif d'enrichissement des formations dégradées en étudiant l'influence des arbres voisins sur la croissance des espèces introduites en plantations d'enrichissement.

\section{MATERIEL ET METHODES Milieu d'étude}

L'étude est conduite dans les formations dégradées de la forêt classée de la Lama en zone guinéenne et des galeries forestières de Bassila en zone soudanoguinéenne. La forêt de la Lama est localisée entre $6^{\circ} 55^{\prime}$ et $6^{\circ} 58^{\prime} \mathrm{N}$ et $2^{\circ} 4^{\prime}$ à $2^{\circ} 10^{\prime} \mathrm{E}$. Les températures moyennes atteignent $35^{\circ} \mathrm{C}$ au mois de février et descendent à $30{ }^{\circ} \mathrm{C}$ en août avec une pluviométrie bimodale de 1.100 mm en moyenne de pluie par an. Les sols de la forêt sont des vertisols de texture argilosableuse comportant une forte teneur en matière organique (Sinsin et al., 2003). Les formations végétales rencontrées sont principalement des forêts denses sèches avec plusieurs niveaux de dégradation (Adomou et al., 2007). Les peuplements enrichis de la Lama sont localisés dans le noyau central de la forêt et sont des forêts denses semidécidues dégradées dans lesquelles sont introduites Terminalia superba, Holoptelea grandis, Khaya grandifoliola et Khaya senegalensis.

La région des galeries forestières de la zone soudano-guinéenne est caractérisée par un régime pluviométrique unimodal. La pluviométrie moyenne annuelle est de 1.200 $\mathrm{mm}$ de pluies réparties d'avril à octobre. Les températures moyennes annuelles varient entre 26 et $27{ }^{\circ} \mathrm{C}$. L'humidité relative est élevée en saison des pluies (juillet-septembre) où elle varie entre 95 et $98 \%$ mais faible en saison sèche avec une valeur de $10 \%$ en janvier et février à cause de l'harmattan qui souffle dans la zone. Les sols de cette région sont en majorité de type ferrugineux tropical (Sokpon et Biaou, 2002). La végétation naturelle de la région est composée de formations forestières denses, de savanes et de galeries forestières. Les peuplements enrichis sont des galeries forestières dégradées présentant différentes physionomies végétales dans lesquelles sont introduites Terminalia superba, Holoptelea grandis, Khaya grandifoliola et Khaya senegalensis.

\section{Dispositif de collecte des données}

Le dispositif de collecte de données est constitué de 8 placeaux carrés de $100 \mathrm{~m}$ de côté qui sont aléatoirement délimités dans des peuplements enrichis du noyau central de la forêt classée de la Lama contre 4 placeaux carrés de $100 \mathrm{~m}$ de côté dans les galeries forestières. A l'intérieur de chacun de ces placeaux, les individus de chacune des espèces introduites sont identifiés et sélectionnés de façon aléatoire sur les lignes de plantations (Tableau 1). Les individus ainsi sélectionnés sont numérotés et marqués à la 
peinture et la distance séparant deux individus sélectionnés est d'au moins $20 \mathrm{~m}$. Enfin, autour de chaque individu sélectionné, est délimitée une placette circulaire de $10 \mathrm{~m}$ de rayon de sorte que deux placettes consécutives n'aient aucun individu en commun (Figure 1). Ainsi, un total de 170 placettes circulaires sont formées dans les peuplements enrichis en zone guinéenne contre 31 placettes circulaires en zone soudano-guinéenne.

Dans chaque placette les données suivantes sont collectées: le nombre de voisins (compétiteurs); la hauteur et le diamètre à 1,3 $\mathrm{m}$ du sol des compétiteurs; la distance séparant chaque voisin de l'individu central. Les mesures prises sur les individus centraux ont été la hauteur totale, le diamètre à $1,3 \mathrm{~m}$ $\mathrm{du}$ sol et le statut social de dominant, de codominant ou de dominé.

\section{Analyse des données}

Afin d'analyser l'influence des individus voisins sur les individus des espèces introduites, une analyse en composantes principales (ACP) est effectuée sur les paramètres dendrométriques des individus voisins situés dans un rayon de $10 \mathrm{~m}$. Cela a permis d'extraire les composantes principales ou axes et de les corréler ensuite avec les paramètres dendrométriques et sociologiques des individus centraux par le test de corrélation de Pearson. Le statut social des individus est codifié 1 pour dominé, 2 pour codominant et 3 pour dominant. Seuls les paramètres dendrométriques ayant des corrélations significatives avec les composantes principales sont pris en compte pour la corrélation avec les paramètres dendrométriques et sociologiques des individus des espèces introduites.

Tableau 1 : Nombres d'individus sélectionnés comme centres de placettes circulaires de $10 \mathrm{~m}$ de rayon selon l'espèce.

\begin{tabular}{lrrrr}
\hline Forêt & H. grandis & K. grandifoliola & K. senegalensis & T. superba \\
\hline FDS & 27 & 45 & 67 & 31 \\
GF & 2 & 15 & 9 & 5 \\
\hline
\end{tabular}

FDS : forêt dense semi-décidue ; GF: galeries forestières.

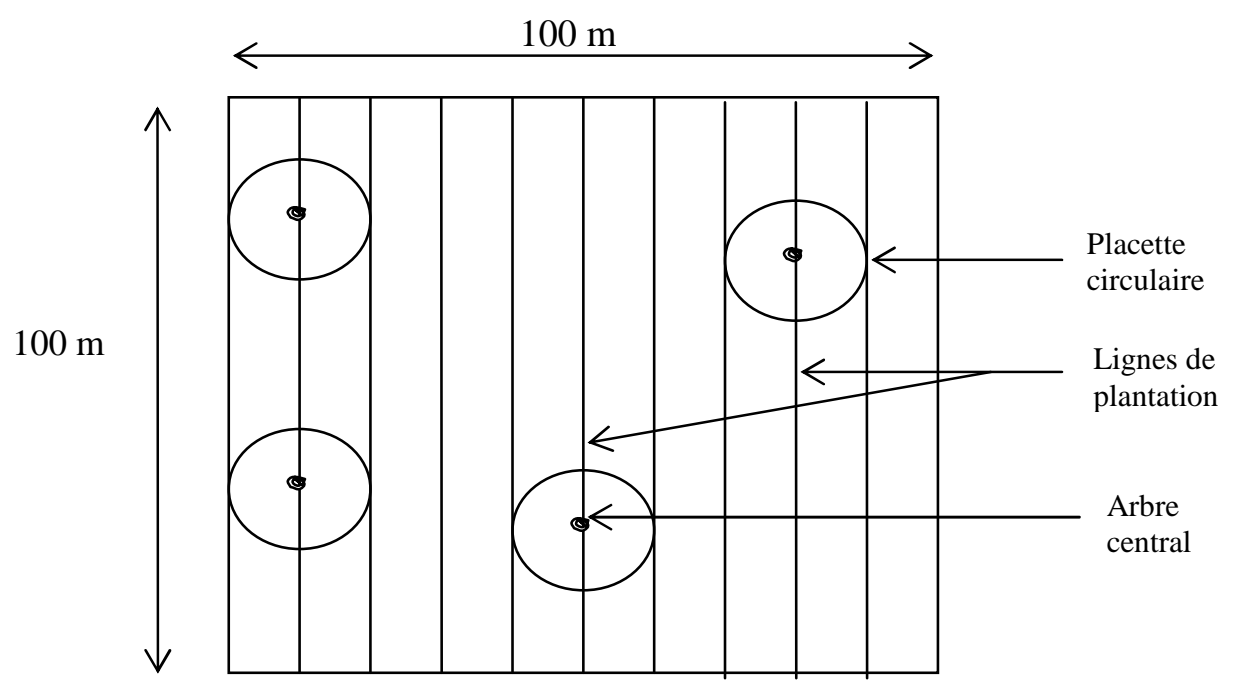

Figure 1: Dispositif d'inventaire relatif à l'étude de l'influence des individus voisins sur les individus des espèces introduites. 


\section{RESULTATS}

Caractéristiques dendrométriques des arbres voisins et centraux

Les caractéristiques dendrométriques des sujets introduits et des arbres voisins ont indiqué une hauteur et un diamètre moyens peu variables d'une espèce introduite à une autre (Tableau 2). Toutefois, les sujets introduits dans les galeries forestières ont été plus vigoureux avec des valeurs de paramètres dendrométriques relativement élevées.

Concernant les arbres voisins ou compétiteurs, leur densité a été plus élevée en zone guinéenne qu'en zone soudanoguinéenne. Néanmoins, les conditions environnementales ont favorisé un développement plus significatif des arbres voisins en galerie forestière en zone soudanoguinéenne qu'en zone guinéenne avec des valeurs de hauteur et diamètre moyens relativement plus élevées en galerie forestière. La distance moyenne de $7 \mathrm{~m}$ entre les individus introduits et les arbres voisins a été pratiquement constante d'une zone climatique à une autre.

\section{Caractéristiques sociologiques des arbres centraux}

Les caractéristiques sociologiques des arbres centraux ont montré qu'environ la moitié des arbres centraux de Khaya senegalensis, de Khaya grandifoliola et de Holoptelea grandis était dominée dans les peuplements enrichis en zone guinéenne alors que la plupart des arbres centraux de Terminalia superba sont dominants ou codominants dans la même zone (Tableau 3). En zone soudano-guinéenne, tous les arbres centraux étaient dominants ou codominants exceptés un arbre central de Khaya grandifoliola et 6 arbres centraux de Khaya senegalensis.

\section{Influence des arbres voisins sur les individus de $\boldsymbol{H}$. grandis}

Les résultats d'analyse en composantes principales effectuée sur les paramètres dendrométriques liés aux arbres voisins de $H$. grandis ont révélé que les trois premiers axes concentraient $97 \%$ de la variabilité globale des paramètres dendrométriques. L'axe $1 \mathrm{a}$ concentré la plupart des caractéristiques dendrométriques des arbres voisins, excepté le diamètre (Tableau 4).

Les axes 2 et 3 par contre, étaient respectivement des axes de grosseur ou de diamètre moyen des arbres voisins et de la distance moyenne séparant les voisins des individus centraux considérés (Tableau 4). Les coefficients de corrélation entre les paramètres dendrométriques des arbres introduits et l'axe 1 ont montré qu'une densité et une hauteur moyenne plus élevées des compétiteurs ou arbres voisins ont induit une hauteur moyenne et un diamètre élevés des individus de $H$. grandis (Tableau 4). Mieux, dans les plantations d'enrichissement de densité et de diamètre moyen élevés des individus voisins, les sujets de $H$. grandis ont été pour la plupart dominés.

\section{Influence des arbres voisins sur les individus de $T$. superba}

Les résultats d'analyse en composantes principales effectuée sur les paramètres dendrométriques liés aux arbres voisins des individus de $T$. superba ont révélé que les trois premiers axes expliquaient respectivement $93 \%$ et $99 \%$ des informations de départ, dans les peuplements enrichis des zones guinéenne et soudano-guinéenne. Ainsi, les trois premiers axes sont retenus au regard de leurs corrélations avec les paramètres dendrométriques des individus centraux de $T$. superba.

En zone guinéenne dans la forêt classée de la Lama, les résultats indiquaient que l'axe 1 concentrait les informations relatives au nombre d'individus voisins et à la distance moyenne séparant ces individus voisins des individus de $T$. superba tandis que l'axe 2 est corrélé au diamètre moyen et à la hauteur moyenne de ces arbres voisins (Tableau 5). Seule la distance moyenne est bien représentée sur l'axe 3. 
Les coefficients de corrélation entre les axes dendrométriques 1 et 2 et les paramètres de $T$. superba montraient que des valeurs élevées de la densité, du diamètre et de la hauteur des individus voisins, associées à de faibles valeurs de la distance moyenne par rapport aux individus centraux de l'espèce, induisaient une croissance significative en hauteur de T. superba (Tableau 5).

En zone soudano-guinéenne dans les galeries forestières de Bassila, la hauteur, le diamètre moyen et la densité des individus voisins sont corrélés au premier axe tandis que le second axe est bien corrélé au nombre d'individus voisins, au diamètre moyen et à la distance les séparant des individus centraux de T. superba (Tableau 5). Seul le diamètre moyen des individus voisins est corrélé à l'axe 3. Les coefficients de corrélation de ces axes dendrométriques avec les caractéristiques dendrométriques et sociologiques de $T$. superba ne montraient aucun lien significatif avec les individus de $T$. superba.

\section{Influence des arbres voisins sur les individus de $K$. senegalensis}

Les trois premiers axes expliquaient $95 \%$ et $98 \%$ des informations sur la variabilité des paramètres dendrométriques liés aux individus autour des individus de $K$. senegalensis, respectivement dans les peuplements enrichis des zones guinéenne et soudano-guinéenne. Ces trois axes sont retenus et interprétés au regard de leurs corrélations avec les paramètres dendrométriques et sociologiques des individus centraux de $K$. senegalensis.

En zone guinéenne, l'axe 1 concentrait les informations relatives aux paramètres à savoir, la hauteur et le diamètre moyens des arbres voisins de $K$. senegalensis dans un rayon de $10 \mathrm{~m}$ (Tableau 6). Les axes 2 et 3 sont corrélés au nombre d'individus voisins et la distance moyenne séparant ces individus des arbres de $K$. senegalensis. L'étude des corrélations entre les axes et les individus de $K$. senegalensis montrait que des plantations d'enrichissement à diamètre moyen et hauteur moyenne élevés des arbres voisins induisaient une croissance significative en hauteur de $K$. senegalensis; par contre les individus de $K$. senegalensis dans ces plantations restaient des individus pour la plupart dominés.

En zone soudano-guinéenne, l'axe 1 est corrélé avec la hauteur et le diamètre moyens des arbres voisins (Tableau 6). Les axes 2 et 3 sont corrélés respectivement à la distance moyenne et à la densité des arbres voisins. L'analyse du signe des corrélations a révélé l'inexistence de liens significatifs entre les paramètres de $K$. senegalensis et ceux des arbres voisins.

Influence des arbres voisins sur les individus de $K$. grandifoliola

Les trois premiers axes expliquaient $93 \%$ et $92 \%$ des informations sur la variabilité des paramètres dendrométriques liés aux individus autour des individus de $K$. grandifoliola, respectivement dans les peuplements enrichis des zones guinéenne et soudano-guinéenne.

En zone guinéenne, l'axe 1 est corrélé au diamètre moyen et à la hauteur moyenne des arbres voisins tandis que les axes 2 et 3 sont corrélés au nombre d'individus voisins et à la distance moyenne (Tableau 7). Les coefficients de corrélation de ces axes dendrométriques avec les individus de $K$. grandifoliola n'indiquaient pas de liens significatifs, excepté le statut social. Dans les plantations d'enrichissement à hauteur et diamètre moyens élevés des arbres voisins, les individus de $K$. grandifoliola étaient pour la plupart dominés. Par ailleurs, l'axe 2 indiquait qu'une densité élevée des arbres voisins induisait une hauteur totale élevée des individus de $K$. grandifoliola.

En zone soudano-guinéenne, tous les paramètres sauf la distance moyenne sont bien représentés sur l'axe 1 . De même, l'axe 2 est corrélé au diamètre moyen des arbres voisins, au nombre d'individus voisins et à la distance moyenne séparant les arbres voisins des individus centraux tandis que l'axe 3 est corrélé à la hauteur moyenne des arbres 
voisins de $K$. grandifoliola. L'analyse des coefficients de corrélation entre ces paramètres et les axes montrait que les plantations d'enrichissement à diamètre moyen et hauteur moyenne élevés des individus voisins induisaient un faible diamètre moyen des individus de $K$. grandifoliola. De plus, l'axe 3 a révélé qu'une hauteur moyenne élevée des arbres voisins induisait une croissance significative en hauteur des individus de $K$. grandifoliola (Tableau 7).

Tableau 2: Moyennes (m) et erreur type de la moyenne (e) des paramètres dendrométriques des peuplements enrichis en zones guinéenne et soudano-guinéenne.

\begin{tabular}{|c|c|c|c|c|c|c|c|c|c|}
\hline & \multicolumn{4}{|c|}{ Zone guinéenne } & \multicolumn{5}{|c|}{ Zone soudano-guinéenne } \\
\hline & & Hg & Kg & Ks & Ts & Hg & Kg & Ks & Ts \\
\hline \multicolumn{10}{|c|}{ Sujets centraux } \\
\hline \multirow{2}{*}{$\mathrm{D}(\mathrm{cm})$} & $\mathrm{m}$ & 13,2 & 14,0 & 14,4 & 19,3 & 27,5 & 25,5 & 18,4 & 24 \\
\hline & es & 0,6 & 0,5 & 0,5 & 1,3 & 7,5 & 1,8 & 2,1 & 2,0 \\
\hline \multirow{2}{*}{$\mathrm{Ht}(\mathrm{m})$} & $\mathrm{m}$ & 11,0 & 10,5 & 8,9 & 12,3 & 20,5 & 18,1 & 10,6 & 18,0 \\
\hline & es & 0,7 & 0,4 & 0,3 & 0,7 & 2,5 & 0,9 & 0,9 & 2,2 \\
\hline \multicolumn{10}{|c|}{ Sujets voisins } \\
\hline \multirow{2}{*}{$\mathrm{NiV}$} & $\mathrm{m}$ & 19,7 & 18,6 & 18,4 & 15,5 & 5,5 & 7,3 & 8,8 & 5,2 \\
\hline & es & 1,5 & 1,0 & 1,0 & 1,6 & 2,5 & 0,8 & 1,3 & 1,1 \\
\hline \multirow{2}{*}{$\begin{array}{l}\text { DiaV } \\
(\mathrm{cm})\end{array}$} & $\mathrm{m}$ & 19,3 & 19,3 & 17,6 & 19,8 & 22,5 & 25,8 & 24,4 & 23,6 \\
\hline & es & 0,7 & 0,5 & 0,4 & 0,8 & 4,5 & 1,4 & 2,3 & 1,8 \\
\hline \multirow{2}{*}{$\mathrm{HtV}(\mathrm{m})$} & $\mathrm{m}$ & 11,8 & 11,4 & 10,2 & 11,3 & 15,0 & 15,3 & 13,1 & 13,6 \\
\hline & es & 0,3 & 0,3 & 0,2 & 0,4 & 1,0 & 0,8 & 0,7 & 1,1 \\
\hline \multirow{2}{*}{ DistV (m) } & $\mathrm{m}$ & 7,1 & 7,3 & 7,1 & 7,3 & 6,5 & 7,2 & 7,0 & 6,8 \\
\hline & es & 0,1 & 0,1 & 0,1 & 0,1 & 0,5 & 0,3 & 0,2 & 0,7 \\
\hline
\end{tabular}

Légende. NiV= nombre d'individus voisins autour de l'arbre central (10 $\mathrm{m}$ de rayon); DiaV $=$ diamètre moyen à $1,3 \mathrm{~m}$ du sol (en $\mathrm{cm}$ ) des individus voisins ; $\mathrm{HtV}=$ hauteur totale moyenne (en $\mathrm{m}$ ) des individus voisins ; DistV= distance moyenne (en $\mathrm{m}$ ) entre les individus voisins et l'arbre central ; $\mathrm{D}=$ diamètre $(\mathrm{en} \mathrm{cm})$ de l'arbre central ; $\mathrm{Ht}=$ hauteur totale $(\mathrm{en} \mathrm{m})$ de l'arbre central ; m : moyenne ajustée; es : erreur standard ; Hg : Holoptelea grandis; Ks: Khaya senegalensis; Kg: Khaya grandifoliola; Ts: Terminalia superba.

Tableau 3: Nombre d'individus centraux dominés, dominants et codominants par espèce des peuplements enrichis en zones guinéenne et soudano-guinéenne.

\begin{tabular}{|c|c|c|c|c|c|c|c|c|}
\hline & \multicolumn{3}{|c|}{ Zone guinéenne } & \multicolumn{5}{|c|}{ Zone soudano-guinéenne } \\
\hline & Hg & Kg & Ks & Ts & Hg & Kg & Ks & Ts \\
\hline $\begin{array}{l}\text { Individus } \\
\text { dominés }\end{array}$ & 16 & 23 & 31 & 8 & 0 & 1 & 6 & 0 \\
\hline $\begin{array}{l}\text { Individus } \\
\text { dominants et } \\
\text { codominants }\end{array}$ & 11 & 22 & 36 & 23 & 2 & 14 & 3 & 5 \\
\hline
\end{tabular}


Tableau 4: Corrélations entre axes dendrométriques des voisins et caractéristiques dendrométriques et sociologiques des individus centraux de $H$. grandis en zone guinéenne.

\begin{tabular}{llrr}
\hline & \multicolumn{3}{c}{ Paramètres liés aux arbres voisins } \\
\hline & Axe1 & Axe2 & Axe3 \\
NiV & 0,86 & $-0,33$ & $-0,32$ \\
DiaV & 0,27 & 0,96 & $-0,03$ \\
HtV & 0,95 & 0,07 & $-0,17$ \\
DistV & 0,53 & $-0,08$ & 0,85
\end{tabular}

Paramètres liés aux individus introduits

\begin{tabular}{llll}
$\mathrm{D}$ & $0,34 * *$ & $-0,28 *$ & $-0,19 \mathrm{~ns}$ \\
$\mathrm{Ht}$ & $0,28^{*}$ & $-0,21 \mathrm{~ns}$ & $-0,22 \mathrm{~ns}$ \\
$\mathrm{Ss}$ & $-0,43 * * *$ & $-0,26 \mathrm{~ns}$ & $0,11 \mathrm{~ns}$ \\
\hline \multicolumn{4}{c}{ *: significatif à 0,$05 ; * *$ * significatif à 0,01} \\
& $* * *$ : significatif à 0,$001 ;$ ns : non significatif à $0,05$.
\end{tabular}

Légende. $\mathrm{NiV}=$ nombre d'individus voisins autour de l'arbre central $(10 \mathrm{~m}$ de rayon); DiaV= diamètre moyen à $1,3 \mathrm{~m}$ du sol (en $\mathrm{cm}$ ) des individus voisins ; $\mathrm{HtV}=$ hauteur totale moyenne (en $\mathrm{m}$ ) des individus voisins ; DistV= distance moyenne (en $\mathrm{m}$ ) entre les individus voisins et l'arbre central ; $\mathrm{D}=$ diamètre $(\mathrm{en} \mathrm{cm})$ de l'arbre central ; $\mathrm{Ht}=$ hauteur totale $(\mathrm{en} \mathrm{m})$ de l'arbre central ; Ss= statut social (dominant, codominant ou dominé) de l'arbre central.

Tableau 5: Corrélations entre axes dendrométriques des voisins et caractéristiques dendrométriques et sociologiques des individus centraux de T. superba.

\begin{tabular}{|c|c|c|c|c|c|c|}
\hline \multirow{2}{*}{$\begin{array}{l}\text { Paramètres liés aux } \\
\text { individus voisins }\end{array}$} & \multicolumn{3}{|c|}{$\begin{array}{l}\text { Peuplements enrichis en zone } \\
\text { guinéenne }\end{array}$} & \multicolumn{3}{|c|}{$\begin{array}{l}\text { Peuplements enrichis en zone } \\
\text { soudano-guinéenne }\end{array}$} \\
\hline & Axe1 & Axe2 & Axe3 & Axe1 & Axe2 & Axe3 \\
\hline $\mathrm{NiV}$ & 0,91 & 0,07 & 0,20 & 0,81 & $-0,57$ & $-0,08$ \\
\hline DiaV & $-0,46$ & 0,75 & $-0,39$ & 0,50 & 0,63 & 0,59 \\
\hline $\mathrm{HtV}$ & 0,44 & 0,83 & 0,21 & $-0,96$ & $-0,12$ & 0,23 \\
\hline DistV & $-0,56$ & 0,13 & 0,81 & 0,04 & 0,90 & $-0,43$ \\
\hline \multicolumn{7}{|c|}{ Paramètres liés aux individus centraux } \\
\hline $\mathrm{D}$ & $0,15 \mathrm{~ns}$ & $0,19 \mathrm{~ns}$ & $-0,23 \mathrm{~ns}$ & $0,59 \mathrm{~ns}$ & $-0,41 \mathrm{~ns}$ & $0,68 \mathrm{~ns}$ \\
\hline $\mathrm{Ht}$ & $0,45^{* *}$ & $0,40^{*}$ & $-0,12 \mathrm{~ns}$ & $-0,61 \mathrm{~ns}$ & $0,70 \mathrm{~ns}$ & $0,03 \mathrm{~ns}$ \\
\hline Ss & $-0,17 \mathrm{~ns}$ & $-0,12 \mathrm{~ns}$ & $-0,14 \mathrm{~ns}$ & $0,20 \mathrm{~ns}$ & $0,82 \mathrm{~ns}$ & $0,08 \mathrm{~ns}$ \\
\hline
\end{tabular}

Légende. $\mathrm{NiV}=$ nombre d'individus voisins autour de l'arbre central (10 $\mathrm{m}$ de rayon) ; DiaV= diamètre moyen à $1,3 \mathrm{~m}$ du sol (en $\mathrm{cm}$ ) des individus voisins ; $\mathrm{HtV}=$ hauteur totale moyenne (en $\mathrm{m}$ ) des individus voisins ; DistV= distance moyenne (en $\mathrm{m}$ ) entre les individus voisins et l'arbre central ; $\mathrm{D}=$ diamètre $(\mathrm{en} \mathrm{cm}$ ) de l'arbre central ; $\mathrm{Ht}=$ hauteur totale (en $\mathrm{m})$ de l'arbre central ; Ss= statut social (dominant, codominant ou dominé) de l'arbre central. 
Tableau 6: Corrélations entre axes dendrométriques des voisins et caractéristiques dendrométriques et sociologiques des individus centraux de K. senegalensis.

\begin{tabular}{lllllll}
\hline \multirow{2}{*}{$\begin{array}{l}\text { Paramètres liés aux } \\
\text { individus voisins }\end{array}$} & \multicolumn{3}{l}{$\begin{array}{l}\text { Peuplements enrichis en zone } \\
\text { guinéenne }\end{array}$} & $\begin{array}{l}\text { Peuplements enrichis en zone } \\
\text { soudano-guinéenne }\end{array}$ \\
\cline { 2 - 7 } & Axe1 & Axe2 & Axe3 & Axe1 & Axe2 & Axe3 \\
\hline $\mathrm{NiV}$ & 0,21 & 0,82 & $-0,52$ & $-0,60$ & $-0,41$ & 0,69 \\
$\mathrm{DiaV}$ & 0,92 & $-0,20$ & 0,12 & 0,86 & 0,32 & 0,34 \\
$\mathrm{HtV}$ & 0,93 & $-0,14$ & $-0,10$ & 0,93 & $-0,11$ & 0,26 \\
$\mathrm{DistV}$ & 0,17 & 0,83 & 0,53 & $-0,53$ & 0,80 & 0,25 \\
& Paramètres liés aux individus centraux & & \\
D & $0,17 \mathrm{~ns}$ & $0,06 \mathrm{~ns}$ & $-0,01 \mathrm{~ns}$ & $-0,11 \mathrm{~ns}$ & $-0,12 \mathrm{~ns}$ & $0,45 \mathrm{~ns}$ \\
$\mathrm{Ht}$ & $0,35^{* *}$ & $0,12 \mathrm{~ns}$ & $-0,10 \mathrm{~ns}$ & $-0,27 \mathrm{~ns}$ & $-0,11 \mathrm{~ns}$ & $0,43 \mathrm{~ns}$ \\
$\mathrm{Ss}$ & $-0,30^{* *}$ & $0,14 \mathrm{~ns}$ & $0,10 \mathrm{~ns}$ & $-0,57 \mathrm{~ns}$ & $0,16 \mathrm{~ns}$ & $-0,23 \mathrm{~ns}$ \\
\hline
\end{tabular}

Légende. $\mathrm{NiV}=$ nombre d'individus voisins autour de l'arbre central $(10 \mathrm{~m}$ de rayon); DiaV= diamètre moyen à $1,3 \mathrm{~m}$ du sol $(\mathrm{en} \mathrm{cm})$ des individus voisins ; $\mathrm{HtV}=$ hauteur totale moyenne (en $\mathrm{m}$ ) des individus voisins ; DistV= distance moyenne (en $\mathrm{m}$ ) entre les individus voisins et l'arbre central; $\mathrm{D}=$ diamètre $(\mathrm{en} \mathrm{cm})$ de l'arbre central; $\mathrm{Ht}=$ hauteur totale $(\mathrm{en} \mathrm{m})$ de l'arbre central ; Ss= statut social (dominant, codominant ou dominé) de l'arbre central.

Tableau 7: Corrélations entre axes dendrométriques des voisins et caractéristiques dendrométriques et sociologiques des individus centraux de $\mathrm{K}$. grandifoliola.

\begin{tabular}{lllllll}
\hline $\begin{array}{l}\text { Paramètres liés aux } \\
\text { individus voisins }\end{array}$ & \multicolumn{3}{l}{$\begin{array}{l}\text { Peuplements enrichis en zone } \\
\text { guinéenne }\end{array}$} & \multicolumn{3}{l}{$\begin{array}{l}\text { Peuplements enrichis en zone } \\
\text { soudano - guinéenne }\end{array}$} \\
\cline { 2 - 7 } & Axe1 & Axe2 & Axe3 & Axe1 & Axe2 & Axe3 \\
\hline $\mathrm{NiV}$ & $-0,32$ & 0,70 & 0,63 & $-0,68$ & 0,56 & 0,41 \\
$\mathrm{DiaV}$ & 0,93 & $-0,02$ & 0,06 & 0,67 & 0,61 & $-0,23$ \\
$\mathrm{HtV}$ & 0,85 & 0,38 & 0,10 & 0,86 & 0,00 & 0,50 \\
$\mathrm{DistV}$ & $-0,10$ & 0,78 & $-0,61$ & $-0,03$ & 0,92 & $-0,10$
\end{tabular}

Paramètres liés aux individus centraux

\begin{tabular}{lllllll}
$\mathrm{D}$ & $0,18 \mathrm{~ns}$ & $0,09 \mathrm{~ns}$ & $0,21 \mathrm{~ns}$ & $-0,52^{*}$ & $-0,15 \mathrm{~ns}$ & $-0,19 \mathrm{~ns}$ \\
$\mathrm{Ht}$ & $0,27 \mathrm{~ns}$ & $0,38^{* *}$ & $0,27 \mathrm{~ns}$ & $-0,10 \mathrm{~ns}$ & $-0,02 \mathrm{~ns}$ & $0,56^{*}$ \\
$\mathrm{Ss}$ & $-0,42^{* *}$ & $0,22 \mathrm{~ns}$ & $-0,08 \mathrm{~ns}$ & $-0,14 \mathrm{~ns}$ & $-0,13 \mathrm{~ns}$ & $-0,04 \mathrm{~ns}$ \\
\hline
\end{tabular}

Légende. $\mathrm{NiV}=$ nombre d'individus voisins autour de l'arbre central $(10 \mathrm{~m}$ de rayon $) ; \mathrm{Dia} V=$ diamètre moyen à $1,3 \mathrm{~m} \mathrm{du}$ sol (en $\mathrm{cm}$ ) des individus voisins ; $\mathrm{HtV}=$ hauteur totale moyenne (en $\mathrm{m}$ ) des individus voisins ; DistV= distance moyenne (en $\mathrm{m})$ entre les individus voisins et l'arbre central ; $\mathrm{D}=$ diamètre $(\mathrm{en} \mathrm{cm})$ de l'arbre central ; $\mathrm{Ht}=$ hauteur totale $(\mathrm{en} \mathrm{m})$ de l'arbre central ; Ss= statut social (dominant, codominant ou dominé) de l'arbre central. 


\section{DISCUSSION}

Caractéristiques dendrométriques et sociologiques des arbres voisins et centraux

En zone guinéenne, les valeurs élevées de hauteur et diamètre moyens de Terminalia superba par rapport aux autres espèces introduites s'expliquent par le fait que cette espèce est à croissance rapide et supporte un ombrage moyen (44\% de lumière). Sa vitesse de croissance est plus élevée que celle des autres espèces introduites (Djodjouwin et Sinsin, 2007). Par ailleurs, la hauteur moyenne des individus centraux de Terminalia superba est plus élevée que celle des arbres voisins autour, ce qui explique que cette espèce est parmi les espèces dominantes ou codominantes des peuplements enrichis dans cette zone. La faible densité des arbres voisins en zone soudano-guinéenne qui explique en partie la vigueur et la bonne croissance des espèces introduites par rapport à celles en zone guinéenne, est due au niveau de dégradation élevé des galeries forestières avant leur enrichissement, au faible écartement des plants introduits (10 m x $10 \mathrm{~m})$ contre $4 \mathrm{~m} \mathrm{x} 4 \mathrm{~m}$ en zone guinéenne et aux conditions du milieu (Agbla, 2009). En effet, la régénération naturelle des ligneux dans les peuplements enrichis en zone guinéenne est plus dynamique qu'en zone soudanoguinéenne, ce qui augmente la densité des voisins en zone guinéenne.

\section{Influence des arbres voisins sur le développement des espèces locales introduites}

La concurrence ou l'influence exercée par les arbres entre eux est le reflet d'un dépassement en hauteur, d'une suppression et d'un encombrement des individus les plus faibles par les individus les plus vigoureux (Rondeux, 1999). La caractérisation des espèces introduites en fonction de l'influence des voisins fait ressortir deux groupes principaux. Le premier groupe est constitué des espèces en grande partie dominées parce que négativement influencées par leurs voisins. Le second groupe est constitué d'une espèce dont l'influence des voisins l'affecte plutôt positivement et est dominante ou codominante dans les peuplements enrichis.
Espèces dominées dans les peuplements enrichis

Holoptelea grandis, Khaya senegalensis et Khaya grandifoliola sont les trois espèces dominées dans les peuplements enrichis. Le nombre de compétiteurs de ces individus centraux, leur diamètre et leur hauteur sont élevés. Ces compétiteurs appartenant à la strate supérieure empêchent les individus centraux d'accéder convenablement à la lumière et réduisent ainsi leurs activités de photosynthèse, ce qui ralentit leur développement en hauteur et en diamètre et fait d'eux des individus dominés de la strate inférieure. Ces résultats sont concordants avec ceux obtenus par Besse (1984) en zone de forêt dense humide de Côte d'Ivoire pour l'espèce Celtis mildbraedii et Agbla (2009) en zone guinéenne du Bénin pour les espèces Khaya senegalensis, Khaya grandifoliola et Afzelia africana. Une observation de la composition spécifique des compétiteurs sur chaque placette montre que Ceiba pentandra, Anogeissus leiocarpa, Lonchocarpus sericeus, Albizia zygia et Diospyros mespiliformis sont les espèces présentes naturellement et les plus importantes du point de vue de la fréquence, de la densité et du recouvrement qui influent négativement sur les espèces dominées. Ces compétiteurs ont des hauteurs pouvant atteindre $20 \mathrm{~m}$ et des diamètres dépassant 60 $\mathrm{cm}$. Ils ont également des couronnes larges créant un ombrage important sur les espèces dominées. Ils sont présents sur chaque placette formée et dominent l'individu central lorsqu'ils sont séparés de lui d'une distance comprise entre 0 et $5 \mathrm{~m}$. En dehors des espèces naturelles présentes, Terminalia superba influe aussi négativement sur les espèces dominées de part sa hauteur élevée, son houppier étalé dès son jeune âge créant ainsi un ombrage sur les individus dominés surtout lorsqu'elle est séparée d'eux d'une distance comprise entre 0 et $5 \mathrm{~m}$. Cette forte influence des espèces naturelles présentes et de Terminalia surperba introduite contribue à la disparition progressive des individus dominés qui sont toutes des espèces de pleine lumière, si rien n'est fait pour modifier la stratification actuelle (Kelly et Cuny, 2000). 
En zone soudano-guinéenne, malgré la densité relativement faible des arbres voisins autour des individus centraux de Khaya seneglensis, cette espèce est maintenue dans la strate inférieure. En effet, Khaya senegalensis est une espèce de pleine lumière supportant mal la concurrence des autres espèces (Agbla, 2009). En ce qui concerne Khaya grandifoliola, cette espèce tolère un certain couvert surtout dans le jeune âge (Dupuy \& Koua, 1993), d'où sa bonne croissance en hauteur. Ainsi, avec la faible densité des compétiteurs, l'espèce a pu émerger dans la strate supérieure en zone soudano-guinéenne. Par contre, l'influence des grands arbres au voisinage empêche le développement de la couronne des individus centraux de Khaya grandifoliola, ce qui réduit leurs activités de photosynthèse limitant ainsi leur croissance en diamètre (Gourlet-Fleury, 1997). Les valeurs calculées des facteurs d'élancement des individus de Khaya grandifoliola sont élevées et supérieures ou égales à 1 , ce qui traduit effectivement une croissance en hauteur nettement plus importante par rapport à sa croissance en diamètre.

\section{Espèce dominante ou codominante}

Terminalia superba est une espèce dominante ou codominante et sa dominance s'explique par sa bonne croissance en hauteur même sous ombrage moyen avec $44 \%$ de passage de lumière. Les caractéristiques biologiques de l'espèce font que l'influence subie de la part de ses compétiteurs stimulerait plutôt sa croissance en hauteur. Par ailleurs, la croissance en diamètre de cette espèce est satisfaisante puisque le facteur d'élancement calculé des individus de Terminalia superba est compris entre 0,60 et 0,75 , ce qui explique un développement harmonieux du diamètre et de la hauteur (Rondeux, 1999). Ainsi, quels que soient le nombre de compétiteurs, la nature des espèces, leur taille, leur grosseur et la distance les séparant des individus centraux de Terminalia superba, ces derniers leur résistent bien avec un bon développement en hauteur et en diamètre.

\section{Conclusion}

Les espèces d'enrichissement introduites dans la forêt classée de la Lama en zone guinéenne et dans les galeries forestières en zone de transition soudano-guinéenne, sont négativement influencées par leurs voisins. Les compétiteurs les plus importants sont ceux qui sont naturellement présents dans les peuplements enrichis et ceci à cause de leur densité, leur forte croissance en hauteur et diamètre et leur large couronne. Les compétiteurs les plus importants sont Ceiba pentandra, Anogeissus leiocarpa, Lonchocarpus sericeus, Albizia zygia et Diospyros mespiliformis pour les forêts denses semi décidues enrichies en zone guinéenne et Berlinia grandiflora, Cola gigantea, Lonchocarpus sericeus, Anogeissus leiocarpa et Pentadesma butyracea pour les galeries forestières enrichies en zone soudanoguinéenne. Les plants de Terminalia superba à croissance rapide en hauteur et leur houppier étalé dans le jeune âge, influent négativement sur la croissance en hauteur des autres espèces introduites. Lorsque ces compétiteurs sont séparés des individus centraux d'une distance comprise entre 0 et $5 \mathrm{~m}$, ils les maintiennent toujours à l'état de dominés.

Holoptelea grandis, Khaya grandifoliola et Khaya senegalensis sont les espèces dominées vulnérables à l'enrichissement. Terminalia superba est la seule espèce introduite dominante dans les peuplements enrichis et à croissance rapide.

Quels que soient les objectifs assignés à ces peuplements enrichis (conservation de la diversité biologique, rétablissement de l'équilibre écologique, promotion des espèces végétales menacées de disparition, production de bois d'œuvre), il est urgent et nécessaire que, sur la base des connaissances actuelles sur les structures des peuplements, les statuts sociaux et la connaissance des influences des voisins sur les individus centraux, soient élaborés et appliqués des plans de gestion simples de ces peuplements enrichis. Ces plans doivent permettre d'identifier et de marquer les arbres d'avenir dans un peuplement final, de modifier au besoin la stratification actuelle des peuplements à travers des éclaircies sélectives pour une 
utilisation optimale des ressources actuelles des peuplements enrichis.

\section{REFERENCES}

Agbla S. 2009. Modélisation des effets de compétition entre arbres et de la structure des zones dégradées de la forêt classée de la Lama enrichies en Afzelia africana Sm, Khaya grandifoliola Welw C.D.C. et Khaya senegalensis Desr. Thèse d'Ingénieur Agronome, Faculté des Sciences Agronomiques, Université d'Abomey - Calavi, Bénin, p. 70.

Adomou AC, Akoegninou A, Sinsin B, De Foucault B, Van der Maesen LGJ. 2007. Biogeographical analysis of the vegetation in Benin. Acta Bot. Gallica, 154(2): 221233.

Besse F. 1984. Contribution à l'étude de l'influence des voisins sur la croissance du BA (Celtis mildbraedii) en forêt de Côte d'Ivoire. Centre Technique Forestier, Nogent- sur - Marne. p.62.

Djodjouwin L, Sinsin B. 2007. Expérience d'aménagement par enrichissement des formations forestières. Actes du $3^{\text {ème }}$ séminaire international de RNSCC, Mayaka T, Sinsin B. (eds). Cotonou, Bénin. 79-96.

Dupuy B. 1992. Les plantations à vocation de bois d'œuvre en forêt dense humide africaine. Bois et Forêts des Tropiques, 231: $5-15$.

Dupuy B, Koua M. 1993. Les plantations d'acajou d'Afrique: leur sylviculture en forêt dense ivoirienne. Bois et Forêts des Tropiques, 236: $26-43$.

Gourley-Fleury S. 1997. Modélisation individuelle spatialement explicite de la dynamique d'un peuplement de forêt dense tropicale humide (dispositif de Paracou-Guyane française). Thèse de doctorat, Université Claude Bernard, Lyon.

Kelly BA, Cuny P. 2000. Plantations d'espèces forestières locales sur sols hydromorphes. Bilan d'une expérimentation sylvicole au sud du Mali. Rev. For. Fr., 5: 453-466.

Nguyen-The N, Favrichon V, Houde L, Fauvet N. 1999. Dynamique de la forêt mixte à diptérocarpacées de basse altitude avant et après traitement sylvicole. Bois et Forêts des Tropiques, 259: 25-44.

Otto HJ. 1997. Les forêts de basse - Saxe en Allemagne du Nord: un passé difficile commande une gestion nouvelle $\left(2^{\text {ème }}\right.$ partie: le programme gouvernemental " Löwe', principes d'une sylviculture proche de la nature). Chronique Internationale : 477 - 488.

Rondeux J. 1999. La Mesure des Peuplements Forestiers. Presses agronomiques de Gembloux: Belgique ; 522 p.

Schmitt L, Barriteau M. 1990. Gestion de l'écosystème forestier guyanais. Etude de la croissance et de la régénération naturelle. Dispositif de Paracou. Bois et Forêts des Tropiques, 220: 3-23.

Sinsin B, Attignon SE, Lachat T, Peveling R, Nagel P. 2003. La forêt de Lama au Bénin: un écosystème menacé sous la loupe. Opuscula Biogeographica Basileensia, 3: 1-32.

Sinsin B, Eyog - Matig O, Sinadouwirou AT, Assogbadjo A. 2002. Caractérisation écologique des essences fourragères Khaya senegalensis et Afzelia africana suivant les gradients de latitude et de station au Bénin. In Development of Appropriate Conservation Strategies for African Forest Countries Identified as Priority Species by SAFORGEN Members Countries, Eyog Matig O, Gaoué OG, Obel - Lawson E (eds). IPGRI: Nairobi, Kenya; $15-50$.

Sokpon N, Biaou SH. 2002. The use of diameter distributions in sustained-use management of remnant forests in Benin: Case of Bassila Forest Reserve in North Benin. Forest Ecology and Management, 161: 13-25. 